\title{
Implantación territorial y análisis arquitectónico de los búnkeres del Subsector IV del estrecho de Gibraltar (Conil, Vejer y Barbate)
}

Territorial implantation and architectural analysis of the bunkers on Subsector IV, Strait of Gibraltar (Conil, Vejer and Barbate)

\author{
Alberto Atanasio-Guisado ${ }^{\text {a }}$, Juan Francisco Molina-Rozalem ${ }^{\text {b }}$ \\ Grupo de Investigación HUM 799 "Estrategias de Conocimiento Patrimonial” - Universidad de Sevilla, Seville, Spain \\ aaag@fidas.org; ${ }^{\text {b }}$ jfmolina@us.es
}

\begin{abstract}
The fortified system executed on the north bank of the Strait of Gibraltar from 1939 pursued two objectives: an offensive one, for which coastal batteries and lighting projectors were installed; and a defensive one, for which around four hundred reinforced concrete bunkers were built for machine guns and / or anti-tank guns along the coastal strip that runs from San Roque to Conil de la Frontera.

According to the military archive documentation, the device for the defense of the land front and against landings on the coast was organized into four subsectors, designated with roman numerals from east to west. Subsector IV, the westernmost, extends from Barbate to Conil, through Vejer de la Frontera. Divided into two resistance centers, it is the one that contained the lowest density of positions, with a total of twenty-seven pillboxes.

This communication has a double purpose. On the one hand, deepen the territorial implantation of the bunker network of Subsector IV, to understand that is fundamental the systemic conception between them and between them and the whole set of bunkers. Secondly, to carry out an individual and specific architectural analysis of each one of the works, focusing on the constructive characteristics and the existence of possible typological relationships.
\end{abstract}

Keywords: Bunkers, Strait of Gibraltar, territorial implantation, architectural analysis.

\section{Introducción}

Al finalizar la Guerra Civil Española se construye en la orilla norte del estrecho de Gibraltar un sistema fortificado con un doble objetivo: recuperar el Peñón de Gibraltar y poder controlar el paso marítimo entre el océano Atlántico y el mar Mediterráneo. Forma parte de esa fortificación el dispositivo para la defensa contra desembarcos en el litoral, compuesto por casi cuatrocientos nidos de hormigón armado preparados para un diferente número de ametralladoras y/o cañones anticarro. Se extendía desde San Roque hasta
Conil de la Frontera, a lo largo de una franja litoral de más de $100 \mathrm{~km}$.

\section{Implantación territorial. El Subsector IV}

El conjunto quedó definitivamente organizado en cuatro subsectores designados con números romanos. El Subsector I desarrollaba sus obras desde la margen izquierda del río Guadiaro (municipio de San Roque) hasta la margen derecha del Guadarranque, límite administrativo entre San Roque y el municipio de Los Barrios. El 
Subsector II comprendía desde el río Guadarranque hasta Punta Carnero, en el extremo occidental de la bahía de Algeciras. El Subsector III incluía todo el término municipal de Tarifa, extendiéndose por sus playas hasta Zahara de los Atunes, localidad ya perteneciente al término de Barbate (Atanasio, 2017).

El Subsector IV, cuyos búnkeres analizaremos a continuación, contaba en 1945 con un total de 27 fortines divididos en dos zonas diferenciadas, A y B. El centro de resistencia IV.A se situaba en la ciudad de Barbate y su ensenada, sin prolongación desde el este, desligándose así de la línea litoral marcada por los búnkeres instalados en Zahara de los Atunes. Se extendía en apenas 5 $\mathrm{km}$ desde la desembocadura del río hasta el borde de la playa de la Yerbabuena, a excepción de los dos fortines dispuestos en sendos flancos de la playa de Caños de Meca (obras IV.A.27 y IV.A.29). La defensa de la ensenada se realizaba según la disposición habitual de la época, con obras más armadas en primera línea y obras en posición más elevada para una sola ametralladora. Con un total de catorce búnkeres, más de la mitad de ellos estaban previstos para 2 ametralladoras.

El centro de resistencia IV.B se desarrollaba en torno a Conil, pero de un modo más extensivo que el anterior, abarcando desde la playa de Zahora hasta cabo Roche. Con un total de 13 obras, la disposición era puntual a lo largo de la playa de El Palmar, cobrando cierta profundidad en el núcleo urbano para proteger tanto el curso del río Salado como el entronque con la carretera de Málaga. Pasado el núcleo urbano hacia el noroeste se vuelve a la disposición de obras puntuales, situadas en salientes de las calas y las playas que llegan hasta cabo Roche (Atanasio, Martínez, 2018) (Fig. 1).

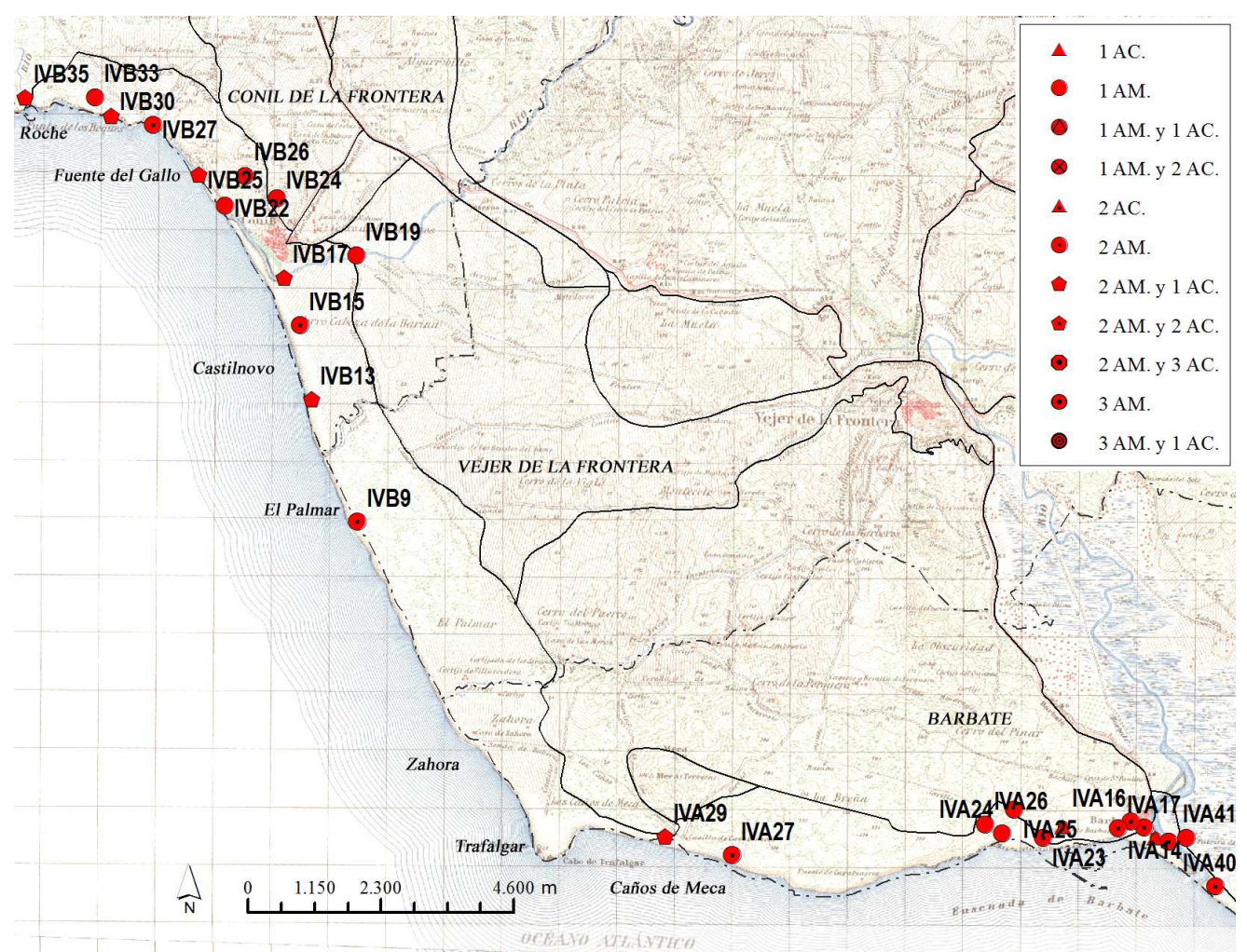

Fig. 1. Implantación territorial del Subsector IV (AM.= Ametralladora; AC.= Anticarro), (Autores, 2017). 


\section{Análisis arquitectónico}

A nivel metodológico, nuestro análisis arquitectónico de los búnkeres del Subsector IV sigue las etapas ya señaladas por el profesor Martínez Medina en su estudio acerca de la arquitectura defensiva ejecutada en la provincia de Alicante durante la Guerra Civil. A saber: escrutinio de revistas y publicaciones de la época, consulta de archivos militares, trabajo de campo para la constatación de la pervivencia de los fortines, y levantamiento de cada una de las obras (Martínez, 2016).

\subsection{Publicaciones}

En los reglamentos militares españoles de los años 20 (Llave García, et al., 1925; Cañellas, 1927) se encuentran recogidos los principios esenciales para el diseño y ejecución de los fortines que aparecerán durante y después de la Guerra Civil Española. Sin embargo, da la sensación de que algunos conceptos no están aún consolidados, inspirados todavía por la fortificación de campaña llevada a cabo durante la Primera Guerra Mundial. Así, en 1925 todavía se conciben separados el puesto de tirador y el abrigo para el personal, aunque se valora ya la posibilidad de unirlos: "El ideal lo constituye un abrigo que sea al mismo tiempo puesto de combate, siendo en este caso la intervención de la tropa instantánea" (Llave García, et al., 1925, p. 88). Resulta una definición precisa de los búnkeres que empezarán a colonizar Europa apenas una década después, aunque en los manuales españoles posteriores a la Guerra Civil no aparezcan todavía los términos búnker o fortín: " [...] bajo el nombre de abrigo se designa tanto la protección elemental que podemos hacer de una trinchera $[\ldots]$; como las grandes obras de hormigón, de varios pisos enterrados que se utilizan en las grandes fortificaciones modernas" (Academia de ingenieros, 1941, p. 83).

Quince o veinte años después, se aprecia una mayor concienciación sobre la necesidad de utilizar el hormigón armado, otorgándole un papel protagonista en la fortificación. La artillería aérea es ahora el referente contra el que defenderse, por lo que se maximiza el principio de diseminación y se prefiere construir muchos abrigos pequeños que pocos abrigos más grandes. A partir de esas premisas, son muchas y muy variadas las posibles configuraciones para un búnker: desde el puesto simple para una ametralladora hasta el tipo propuesto por Franco, "trinchera de hormigón y acero", más amplio que la mayoría de diseños de la época (Franco, 1944, pp. 31-32) (Figs. 2-4).

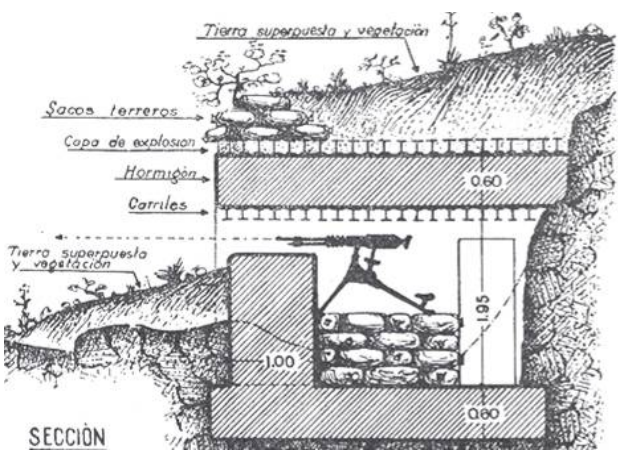

Fig. 2. Fortín para una ametralladora (Capdevila, 1939, p. 186).

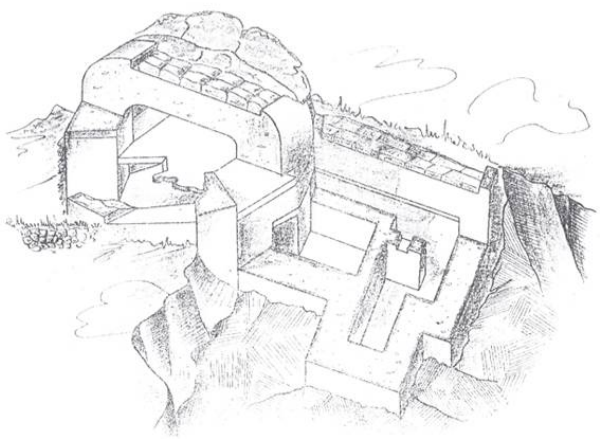

Fig. 3. Fortín para una ametralladora con sector de tiro a $360^{\circ}$, refugio y municiones (Academia, 1941, s/p).

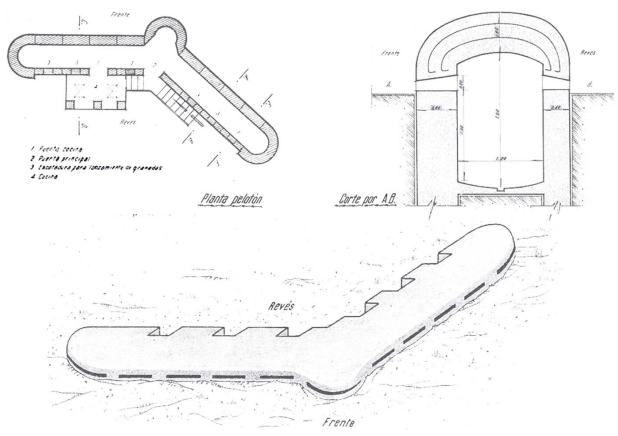

Fig. 4. Fortín para pelotón (Franco, 1944, s/p). 


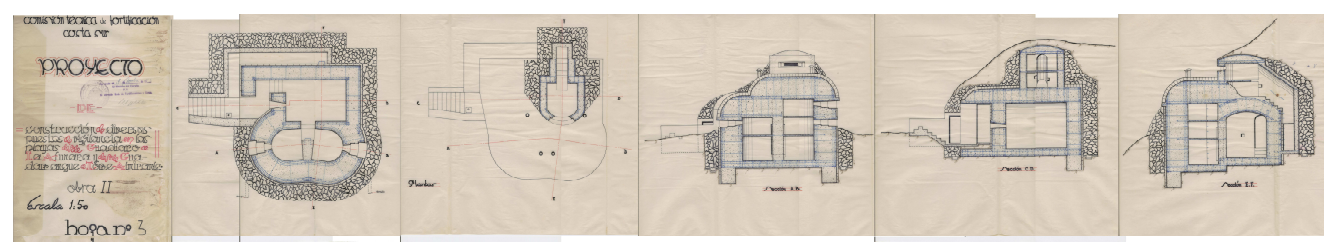

Fig. 5. Planimetría de la obra $\mathrm{n}^{\mathrm{o}} 2 \mathrm{del}$ "Proyecto para la construcción de diversos puestos de vigilancia en las playas desde Guadiaro a la Atunara y desde Guadarranque a Torre Almirante". De los ocho búnkeres proyectados, sólo dos guardan cierta relación entre sí (AIMSS, 1943, plano 19/58).

\subsection{Material de archivo}

Los búnkeres de la orilla norte del Estrecho no se construyeron a partir de un único proyecto. Es probable que existieran unas premisas básicas sobre localización y características principales, pero los emplazamientos definitivos y el diseño de las obras fueron trazados por los mandos tácticos de cada Subsector, quienes se encargaron de redactar los proyectos correspondientes. Esto explicaría las diferencias presentes entre obras de un extremo $u$ otro, ya anunciadas en el informe de inspección cursado por la Dirección General de Obras en 1940, que aludía al excesivo tamaño de los nidos de la playa de Los Lances de Tarifa frente a la idoneidad de los de la bahía de Algeciras. ${ }^{1}$

En el Archivo Intermedio Militar Sur de Sevilla (AIMSS) pudimos localizar un buen número de esos proyectos, compuestos al modo de los documentos actuales por memoria, planimetría y mediciones. La variedad de los tipos propuestos es absoluta, y en pocas ocasiones se repite un modelo dentro del mismo proyecto, previstos normalmente para la construcción de un número limitado de unidades (Fig. 5).

El más numeroso de todos ellos es el "Proyecto de construcción de 58 obras de campaña correspondientes al plan defensivo del Campo de Gibraltar", del cual hallamos íntegras la memoria y las mediciones, pero sólo parte de la planimetría. De los cuatro planos de situación que debería recoger el Proyecto únicamente pudimos encontrar dos, y de las cincuenta y ocho figuras de búnkeres sólo diecinueve, todas pertenecientes a los Subsectores III y IV. La memoria del proyecto recoge una síntesis tipo de las características técnicas de todos los fortines proyectados. He- mos querido transcribir el texto, dado que se trata de una muestra bastante representativa:

"Todas las obras proyectadas lo han sido con arreglo a unas características generales y normas comunes, que fundamentalmente pueden resumirse en: Parte activa: cámara de tiro, puestos de granaderos, pozos de gola y galería para fusileros a cielo abierto, cubiertas de las vistas. Construcción enterrada todo lo posible, para conseguir una mayor ocultación de la obra y facilitar el enmascaramiento de la parte que forzosamente emerja del terreno, cuidando que éstas no se proyecten en ningún caso recortadas en el horizonte; la parte enterrada tiene así un aumento de protección y además permite lograr la mayor rasancia en el fuego, que es evidentemente el empleo peculiar de las armas automáticas y el tiro de su máximo rendimiento. Clase de fábrica a emplear: hormigón en masa o ciclópeo en cimientos [...]; muros, de hormigón en masa con espesores de 0,60 a 1,00 metros y losas de blindaje de hormigón de $400 \mathrm{~kg}$ de cemento, con espesores de 0,40 a 1,00 metros, armado con 1, 2 ó 3 emparrillados de hierro redondo, según las luces, de 6 a $20 \mathrm{~mm}$ de diámetro [...]. Parte pasiva: Repuestos para municiones y alojamientos para el personal. Estos están totalmente enterrados y tienen las dimensiones mínimas, pensando en el empleo de camas-literas. Clase de fábrica a emplear: hormigón en masa, aunque en casos excepcionales tiene también algunos muros de mampostería, espesores de 0,40 a 0,60 metros; losas de blindaje de hormigón de $400 \mathrm{~kg}$ de cemento, con espesores de 0,40 a 0,60 metros, armado con 1 ó 2 emparrillados de hierro redondo, según las luces, de 6 a $20 \mathrm{~mm}$ de diámetro $[\ldots] "{ }^{2}$ 


\subsection{Trabajo de campo y levantamientos}

Sigan existiendo o hayan desaparecido, a través de la planimetría original o el trabajo de campo se ha podido realizar el levantamiento de la mayoría de los fortines ejecutados en el Subsector IV. De un total de veintisiete obras se han representado veinte: siete para 1 ametralladora, siete para 2 ametralladoras, dos para 1 cañón anticarro, y cuatro para 2 ametralladoras y 1 anticarro. El grado de fiabilidad de cada levantamiento es variable, ya sea porque se ha realizado sobre plano (está fotografiado, no escaneado, con la pérdida de escala que ello puede acarrear), ya sea porque sobre el terreno o el propio búnker presentaban algún tipo de riesgo o dificultad (Fig. 6).

\begin{tabular}{|c|c|c|c|c|}
\hline Mun. & Ref. & Armas & Plan orig & Levantamiento \\
\hline \multirow{14}{*}{ 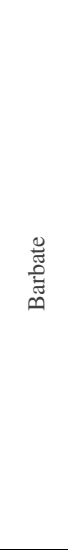 } & IVA13 & $2 \mathrm{AM}$ & No & Desaparecido \\
\hline & IVA14 & $2 \mathrm{AM}$ & No & Desaparecido \\
\hline & IVA14b & $1 \mathrm{AC}$ & $\mathrm{Si}$ & P58 \\
\hline & IVA16 & $2 \mathrm{AM}$ & No & Desaparecido \\
\hline & IVA17* & $2 \mathrm{AM}$ & No & Desaparecido \\
\hline & IVA21 & $1 \mathrm{AC}$ & $\mathrm{Si}$ & P58 \\
\hline & IVA23 & $2 \mathrm{AM}$ & $\mathrm{Si}$ & P58 \\
\hline & IVA24 & $1 \mathrm{AM} / 2 \mathrm{AM}$ & $\mathrm{Si}$ & P58 \\
\hline & IVA25 & $1 \mathrm{AM}$ & No & Exterior \\
\hline & IVA26 & $1 \mathrm{AM}$ & No & Completo \\
\hline & IVA40 & $2 \mathrm{AM}$ & No & Completo \\
\hline & IVA41 & $1 \mathrm{AM}$ & No & Completo \\
\hline & IVA27 & $2 \mathrm{AM}$ & $\mathrm{Si}$ & Ext./P58 \\
\hline & IVA29 & $2 \mathrm{AM}+1 \mathrm{AC}$ & No & Inaccesible \\
\hline Vejer & IVB9 & $2 \mathrm{AM}$ & $\mathrm{Si}$ & Completo \\
\hline \multirow{12}{*}{ 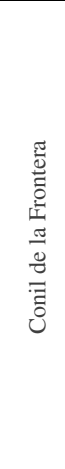 } & IVB13 & $2 \mathrm{AM}+1 \mathrm{AC}$ & No & Completo \\
\hline & IVB15 & $2 \mathrm{AM}$ & $\mathrm{Si}$ & Completo \\
\hline & IVB17 & $2 \mathrm{AM}+1 \mathrm{AC}$ & $\mathrm{Si}$ & P58 \\
\hline & IVB19 & $1 \mathrm{AM}$ & No & Foto aérea \\
\hline & IVB22 & $1 \mathrm{AM}$ & No & Desaparecido \\
\hline & IVB24 & $1 \mathrm{AM}$ & $\mathrm{Si}$ & P58 \\
\hline & IVB25 & $2 \mathrm{AM}+1 \mathrm{AC}$ & $\mathrm{Si}$ & Exterior/P58 \\
\hline & IVB26 & $1 \mathrm{AM}$ & $\mathrm{Si}$ & Completo \\
\hline & IVB27 & $2 \mathrm{AM}$ & No & Int./Foto aérea \\
\hline & IVB30 & $2 \mathrm{AM}+1 \mathrm{AC}$ & No & Desaparecido \\
\hline & IVB33 & $1 \mathrm{AM}$ & No & Ext./Fotografía \\
\hline & IVB35 & $2 \mathrm{AM}+1 \mathrm{AC}$ & $\mathrm{Si}$ & P58 \\
\hline
\end{tabular}

Fig. 6. Tabla resumen. En la columna izquierda, en verde, los búnkeres localizados; en la columna derecha, en verde, aquéllos que han podido ser representados (Autores, 2017).
Observando la proyección en planta de los fortines inventariados, resultan comunes en todos ellos una serie de piezas:

a) El tambor de tiro es la estancia delantera, donde se colocaba el arma y, por tanto, donde se practicaban las aspilleras. Siempre de directriz frontal curva, en ocasiones el muro de hormigón delantero presenta mayor espesor que los muros de estancias posteriores.

b) El depósito de municiones suele ser una estancia intermedia, la más cercana al armamento y de menor tamaño dentro del búnker. En las obras preparadas para 1 ametralladora se configura más como un nicho o un armario que como una verdadera estancia.

c) El refugio o zona de alojamiento, normalmente en la parte posterior del fortín y, en ocasiones, con acceso propio desde el exterior.

d) La galería interior y el ramal de acceso. En parte cubiertos y en parte descubiertos, estos ramales perseguían desenfilar el acceso a los búnkeres del posible fuego enemigo. Las obras previstas para la acción de cañones anticarro presentan una galería de acceso de anchura considerable, mucho mayor que las previstas solo para ametralladoras. Frente al resto de búnkeres del Campo de Gibraltar, se observa en el Subsector IV un mayor desarrollo de los ramales, tanto en su longitud como en su número, especialmente en la zona de Conil.

e) Pozos de tiradores. Se trata de pozos descubiertos, situados normalmente en los extremos posteriores de los ramales de acceso para defender la gola o retaguardia. En las obras de mayor envergadura aparecen también los pozos para granaderos, adosados en los muros laterales del fortín, con acceso cubierto desde el interior.

En cualquier caso, y a pesar de que efectivamente todos los búnkeres responden de un modo u otro a esta distribución, no puede decirse que los del Subsector IV sean producto de una rigurosa sistematización de las obras. Más bien al contrario: es cierto que existen ciertas correspondencias tipológicas, pero los fortines destacan más por su diversidad que por mantener patrones homogéneos (Fig. 7). 

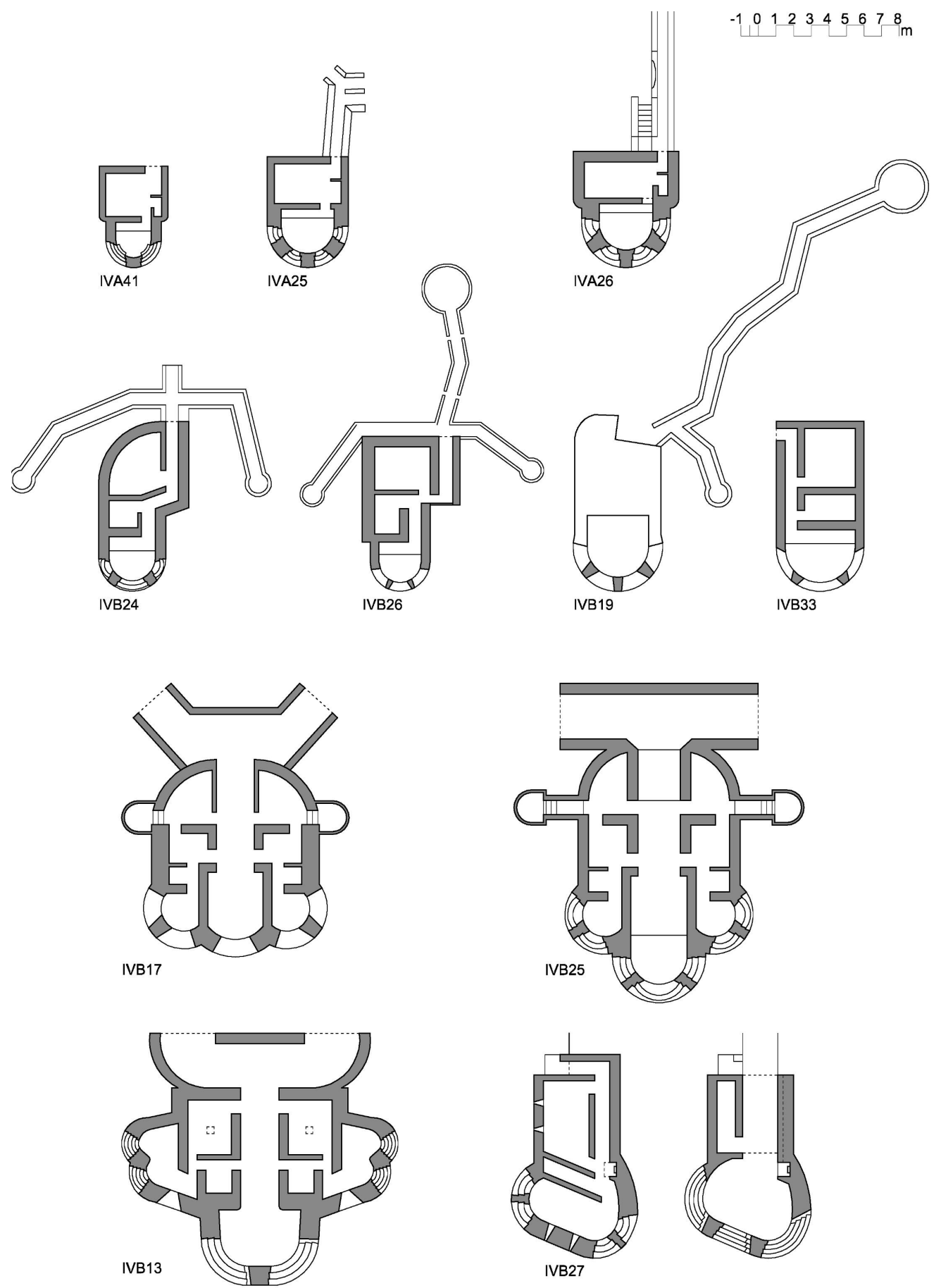

Fig. 7. Primera fila, obras para 1 ametralladora en Barbate. Segunda fila, ídem en Conil. Tercera fila, obras para 2 ametralladoras y 1 anticarro en Barbate. Cuarta fila, ídem en Conil. Los fortines de las tres primeras filas tienen correspondencia tipológica entre ellos pero no con el resto. Las de la última fila son únicos (Autores, 2017). 
Las imágenes tridimensionales obtenidas de los levantamientos no pretenden en absoluto ser realistas. No sería de rigor teniendo en cuenta el poco detalle de la planimetría de referencia, en donde se ha detectado, por ejemplo, que no se representan los desniveles propios del terreno de emplazamiento. La intención de estas vistas no es otra sino la de tomar contacto con el objeto arquitectónico, conocer su distribución, sus características esenciales. Los acabados se representan por lo general con color blanco para el cemento o el hormigón, acaso con algunas texturas poco definidas si se trata de búnkeres todavía existentes en donde pueda leerse claramente el material de revestimiento (Fig. 8).

\section{Conclusiones}

El Subsector IV es el de menor densidad de obras de todo el sistema contra el desembarco en el litoral ejecutado en la orilla norte del Estrecho. Según la documentación del AIMSS, en 1945 se habían ejecutado 27 búnkeres entre Barbate, Vejer y Conil (Atanasio, 2017). De esos 27 se han localizado por el momento 15 , pero gracias a la planimetría de archivo hemos podido esbozar el levantamiento de 20 de ellos. A pri- mera vista, las obras mantienen una serie de características comunes, dando cumplimiento con mayor o menor fidelidad a los principios establecidos en los reglamentos y manuales de la época: se construyen en hormigón armado, se revisten por una capa de explosión, quedan compartimentadas en las estancias necesarias y suficientes, etc. Sin embargo, la formalización de todos esos principios no permite establecer de manera definitiva un patrón tipológico, ni con otros dispositivos fortificados (costa occidental de Cádiz, Alicante, etc.), ni con los fortines pertenecientes a otros subsectores dentro del propio dispositivo. De hecho, tanta es la diversidad de obras que ni siquiera hay correspondencia alguna entre los búnkeres de Conil y los búnkeres de Barbate.

Ahí radica la complejidad -y también la riqueza- de gran parte de la fortificación del siglo $\mathrm{XX}$, en donde un búnker puede encarnar un ejemplar único a la vez que resulta componente indispensable de un sistema disperso y fragmentado. Es tarea pendiente inventariar y clasificar cada uno de esos componentes, para así mejor conocer y comprender el sistema desde cada una de sus partes.
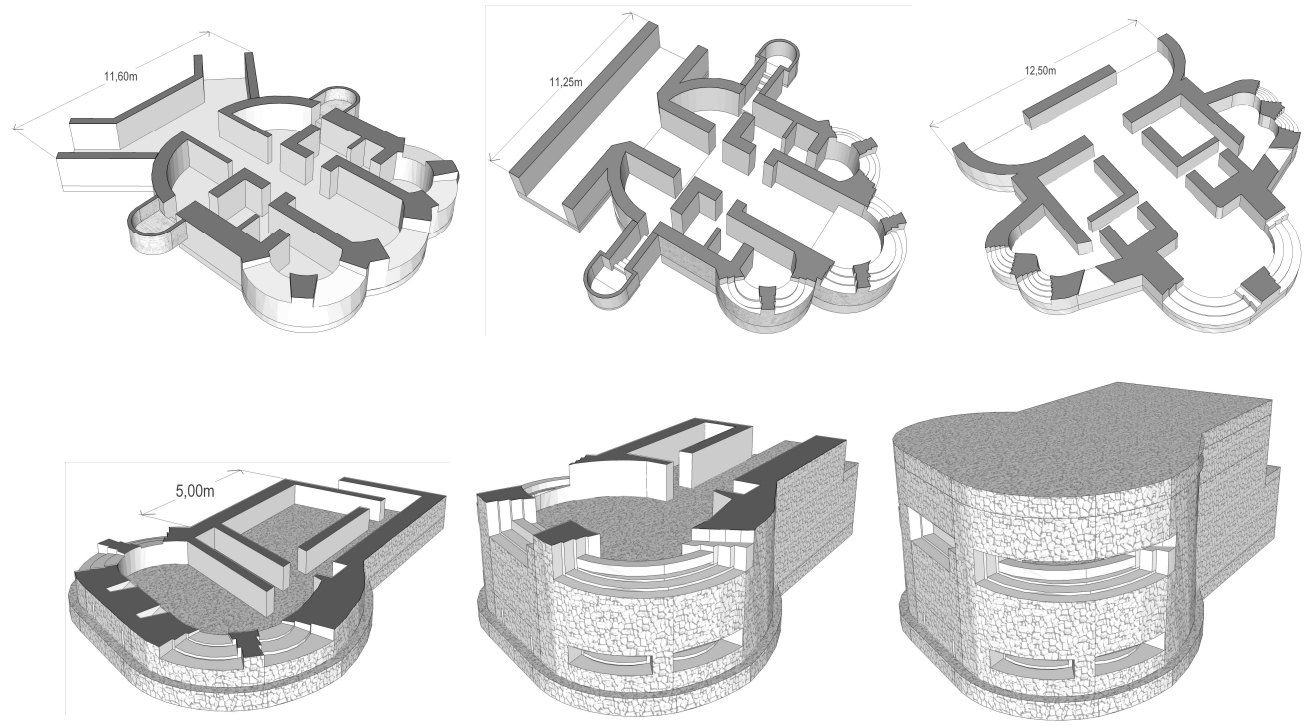

Fig. 8. Cuatro búnkeres para 2 ametralladoras y 1 anticarro en Conil. Arriba, de izquierda a derecha, el IV.B.17, el IV.B.25 y el IV.B.13. Abajo, dos plantas y cubierta del IV.B.27 (Autores, 2017). 


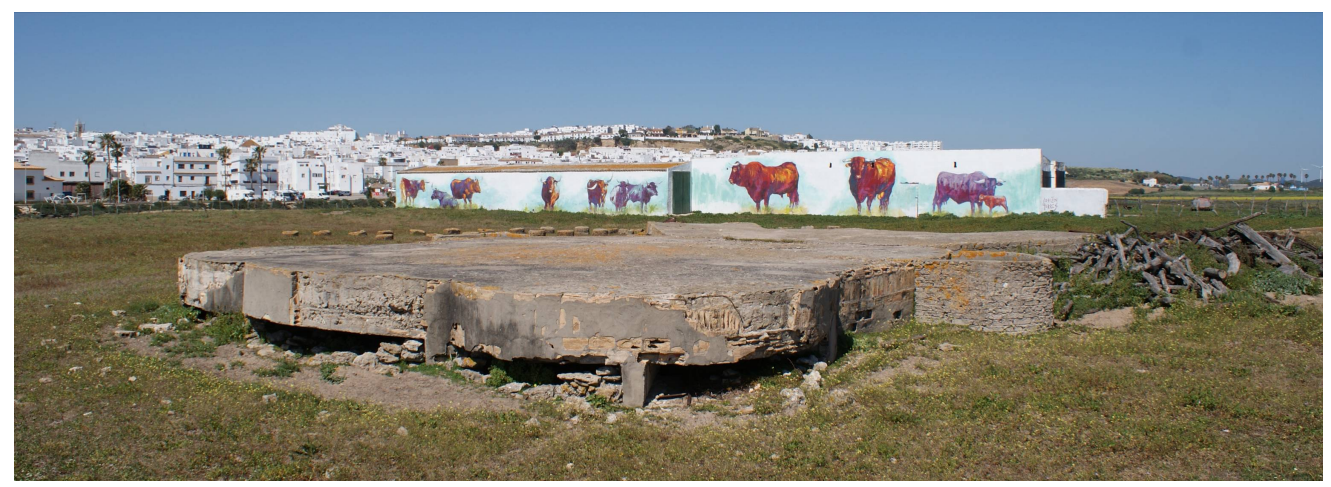

Fig. 9. El búnker IV.B.17, en una finca privada junto al puente del río Salado, Conil de la Frontera (Autor, 2017).

\section{Notas}

${ }^{1}$ Dirección General de Fortificaciones y Obras (1940). "Inspección de la Dirección General de Fortificaciones y obras", Archivo Municipal de Cádiz, Fondo Varela, VT 98-20 a 98-24.

2“Proyecto de construcción de 58 Obras de campaña correspondientes al plan defensivo del Campo de Gibraltar" (1944). Archivo Intermedio Militar Sur de Sevilla. Memoria en signatura 3981 , carpeta 6, planos 17/54 a 17/60. Actualmente, los fondos han sido transferidos al Archivo General Militar de Ávila.

\section{Agradecimientos}

Esta investigación forma parte del proyecto HAR2016-78113-R del Programa Nacional de Investigación, Desarrollo e Innovación dirigido a los Retos de la Sociedad, financiado por el $\mathrm{M}^{\circ}$ de Economía y Competitividad, y los FEDER.

\section{Bibliography}

Academia de ingenieros. (1941). Fortificación, $1^{\circ}$ grupo, $3^{\circ}$ curso, Imprenta Aldecoa, Burgos.

Atanasio Guisado, A. (2017). Arquitectura defensiva del siglo XX en el Campo de Gibraltar. Implantación territorial, análisis tipológico y valor patrimonial de los búnkeres, Tesis doctoral (inédita), Universidad de Sevilla, Sevilla.

Atanasio Guisado, A.; Martínez Medina, A. (2018). "Fortificación del siglo XX en la orilla norte del estrecho de Gibraltar", en Marotta, A.; Spallone, R., eds., FORTMED 2018. Defensive architecture of the Mediterranean. Proceedings of the International Conference on Modern Age Fortification of the Mediterranean Coast, Politecnico di Torino, Torino, vol. IX, pp. 1357-1364.

Cañellas Marquina, L.; et al. (1927). Reglamento para la organización y preparación del terreno para el combate, Talleres del Depósito de la Guerra, Madrid.

Capdevila, J. (1939). Fortificación de campaña, Sindicato de Industria, Barcelona.

Franco Bahamonde, F. (1944). ABC de la batalla defensiva, Imprenta del Servicio Geográfico del Ejército, Madrid.

Llave García, J. de la; et al. (1925). Reglamento para la instrucción técnica relativa a los trabajos de fortificación y castrametación propios de las tropas de zapadores minadores, Estado Mayor Central del Ejército, Imprenta Cervantina, Madrid.

Martínez Medina, A. (2016). Arquitecturas para la defensa de la costa Mediterránea (1936-1939), Catálogo de la exposición, Universidad de Alicante. 